\title{
Biotechnological approaches for enhancing animal health and production
}

\begin{abstract}
A field survey was conducted to collect the first-hand information on the constraints being perceived by buffalo owners in Jaipur district of Rajasthan. The data were collected from 160 buffalo rearers randomly selected from two tehsils of Jaipur district by means of pretested and pre-designed interview schedule. As inferred from a pooled RBQ analysis, the farmers' negligence in vaccinating the animals was one of the main constraints faced by buffalo owners. The RBQ value of this constraint was 62.12 and the constraint was ranked first in both Amber and Phulera tehsil. Repeat breeders were ranked as the second most serious constraint, with the RBQ value of 57.75 on pooled RBQ basis. Based on distribution of RBQ values, this constraint was ranked second in both Phulera and Amber tehsils. Lack of AI centers, poorly equipped AI centers, and their poor service were the third most serious constraint in the area surveyed, with the RBQ value of 54.68. This constraint ranked third in Amber and fifth in Phulera tehsil, respectively.
\end{abstract}

Keywords: survey, buffaloes, RBQ, constraint, repeat breeder
Volume 2 Issue I - 2015

\author{
Amrutlal K Patel,' Mahavir Singh ${ }^{2}$ \\ 'Department of Animal Biotechnology, Veterinary College, India \\ ${ }^{2}$ Department of Medicine, University of Louisville, USA
}

\begin{abstract}
Correspondence: Mahavir Singh, Department of Medicine, University of Louisville, Louisville, Kentucky 4024I, USA, Tel (502) 852 2477, Fax (502) 8523663 ,
\end{abstract}

Email mahavir.singh@louisville.edu

Received: March 05, 2015 | Published: March 09, 2015
Abbreviations: POC, point of care; NGS, next generation sequencing; MAS, marker assisted selection

\section{Editorial}

The domestication of livestock has played crucial role in the evolution of human civilization as well as in shaping human genome through generating selection pressure to metabolize the animal derived food products. Livestock production has been the sustainable source of income for farmers and contributes significantly to the world economy. The demand for livestock products has been increasing due to the global rise in human population which is estimated to be nearly 10billion by $2050 .{ }^{1}$ However due to urbanization and reduction in the natural resources, the cost of livestock production has also substantially increased. To sustain the livestock production and meet the global increase in the demand of livestock products, numerous attempts are being made to enhance the animal productivity and health. Among the various approaches, biotechnological approaches which are briefly summed up here offer promise to enhance livestock health and production.

Improvement of livestock has traditionally focused on the selective breeding of individuals with superior phenotypes to allow them to perform better as well as survive and thrive in the prevailing environment. This approach has been extremely successful in increasing the quantity of agricultural output, however, recent advances in genetic technologies which not only enabled identification of genetic markers associated with the health and production traits but also led to the information of their entire genome. Marker assisted selection (MAS) which offers several merits over conventional phenotypic selection, has been integrated in the breeding program for genetic improvement of livestock. MAS strategy targets one or few alleles governing the particular traits thereby resulting in the selection of individuals with superior alleles. This strategy results in a small number of individuals having a large influence on the gene pool which in turn results in the loss of genetic diversity. Recently, genomic selection which involves selection for numerous genetic markers dispersed across the genome contributing to multiple traits has been seen as an ideal approach to estimate the breeding value as well as maintaining the genetic diversity. ${ }^{2}$

Ruminants comprise major livestock species among the domesticated animals. Ruminants harbors complex microbial communities in their fore stomach known as rumen which are essential for conversion of coarse feed into digestible compounds such as volatile fatty acids and bacterial proteins, which in-turn determines the animal health and productivity (Pope et al., 2012). The rumen microbial community providing such beneficial functions is dominated by bacteria followed by anaerobic protozoa, fungi and bacteriophages. ${ }^{3}$ Recently, microbiome dynamics in response to change in the diet suggested significant impact of diet on rumen microbiome. ${ }^{4}$ These microbial dynamics suggested key role played by specific microbial communities in the degradation of fiber rich diet. Among the all rumen microbial species, less than $11 \%$ are cultivable. ${ }^{5}$ The availability of complete genome sequence of rumen microbial communities would further facilitates devising culture media for supporting the culture of these microbial species. In the near future, we hope that many of these species may hold potential to be used as probiotics to enhance digestibility and thereby production.

The ability of rumen microflora to degrade the coarse feed is ascribed to their ligno-cellulolytic enzymes. These enzymes have evolved with their host depending on their feeding habitat. The ruminants are known for efficient conversion of lignocellulosic biomass to metabolic energy and hence expected to harbor enzymes with high potency so as to degrade the coarse plant fibers in few hours. These enzymes are useful for number of applications such as animal feed supplements to enhance the digestibility, for production of biofuel, fermentation industry, pulp and paper industry, textile industry, food and feed industry and production of other value-added commodities. $^{6-8}$ The mining of cellulolytic enzyme from rumen microbiome has the potential discover novel biocatalyst with high potency. 
Assisted reproductive technologies including embryo transfer, somatic cell nuclear transfer and transgenesis have been seen as promising approaches to introduce the novel and superior traits in the livestock such as high production traits, disease resistance, and production of biopharmaceuticals. However, these approaches require skilled manpower and expensive equipments. Further, limited success and ethical issues concerning genetically modified animals have restricted these technologies to the laboratory.

The recent advancement in molecular biology has also led to the development of diagnostic and therapeutic tools to improve the animal health. Accurate and timely diagnosis of infectious diseases is not only essential to reduce the morbidity and mortality associated with them but also necessary to reduce the use of antibiotics and to halt the development of antibiotic resistance. Conventional diagnostic tests based on the microscopic examination, classical bacterial culture methods and the results obtained via biochemical analysis are relatively less sensitive, and require several days to obtain results. Further, they are also limited to the cultivable properties of the respective microorganisms. The development of molecular assays will help facilitate the culture independent and rapid diagnosis of infectious agents with high sensitivity and specificity as well as will enable the identification of antibiotic resistant genes. Although, molecular tools holds promise for development of point of care (POC) diagnostics options, we are yet to witness such assays which can simultaneously screen multiple pathogens and antibiotic resistant markers in order to help clinicians to determine the best possible line of treatment options. Among the various molecular diagnostics, isothermal nucleic acid amplification techniques ${ }^{9}$ such as loop mediated isothermal amplification and helicase dependent amplification appear most practical solution to develop rapid, simple, affordable and portable diagnostic options without compromising sensitivity and specificity whereas next generation sequencing (NGS) platforms which are still expensive to be adopted in routine clinical practice, offer feasibility to rapidly identify the novel and emerging pathogens for diagnosis of diseases with unknown etiology. Further advances in the microfluidic devices combined with the bio-recognition and electrochemical detection systems would facilitate development of portable point of care biosensors for sensitive and rapid diagnosis of infections. ${ }^{10}$

Finally, the development of recombinant vaccines is one of the major advancements in biotechnology for the prevention of a number of viral and bacterial diseases. These vaccines include DNA vaccines, vectored vaccines, and subunit vaccines which offer increased safety over traditional inactivated or killed vaccines. Interestingly, among other therapeutic modalities such as monoclonal antibodies, phage therapy, RNA interference, interferons, gene therapy and stem cells have been emerging in the human medicines and are likely to improve the available treatment options in the veterinary medicines as well.

\section{Acknowledgements}

None.

\section{Conflict of interest}

Author declares that there is no conflict of interest.

\section{References}

1. Thornton PK. Livestock production: recent trends, future prospects. $P h i$ los Trans R Soc Lond B Biol Sci. 2010;365(1554):2853-2867.

2. Hayes BJ, Bowman PJ, Chamberlain AJ, et al. Invited review: Genomic selection in dairy cattle: progress and challenges. J Dairy Sci. 2009;92(2):433-443.

3. Brulc JM, Antonopoulos DA, Miller ME, et al. Gene-centric metagenomics of the fiber-adherent bovine rumen microbiome reveals forage specific glycoside hydrolases. Proc Natl Acad Sci USA. 2009;106(6):1948-1953.

4. Parmar NR, Solanki JV, Patel AB, et al. Metagenome of Mehsani buffalo rumen microbiota: an assessment of variation in feed-dependent phylogenetic and functional classification. J Mol Microbiol Biotechnol. 2014;24(4):249-261.

5. Edwards JE, Huws SA, Kim EJ, et al. Characterization of the dynamics of initial bacterial colonization of nonconserved forage in the bovine rumen. FEMS Microbiol Ecol. 2007;62(3):323-335.

6. Ikeda Y, Park EY, Okuda N. Bioconversion of waste office paper to gluconic acid in a turbine blade reactor by the filamentous fungus Aspergillus niger. Bioresour Technol. 2006;97(8):1030-1035.

7. Kuhad RC, Gupta R, Singh A. Microbial cellulases and their industrial applications. Enzyme Res. 2011.

8. Wen Z, Liao W, Chen S. Production of cellulase by Trichoderma reesei from dairy manure. Bioresour Technol. 2005;96(4):491-499

9. Craw P, Balachandran W. Isothermal nucleic acid amplification technologies for point-of-care diagnostics: a critical review. Lab Chip. 2012;12(14):2469-2486.

10. Ahmed MU, Hossain MM, Safavieh M, et al. Toward the development of smart and low cost point-of-care biosensors based on screen printed electrodes. Crit Rev Biotechnol. 2015;12:1-11. 